\title{
Dead Set
}

\author{
Maree Collie
}

They arrived late, those nameless men, to prepare the garage floor for concrete. They were supposed to come last week. They packed down the soil. It was loose they said. They shovelled in the gravel and screenings, packed that down too. The cement came, flowed in and was levelled.

He won't have to complain, ever again, that I disturb him. 